\title{
Music in Advertising and Research Methods in Psychology
}

\author{
Yi Ji \\ Music and Dance College \\ Qujing Normal University \\ Qujing, Yunnan, China 655011
}

\begin{abstract}
Music not only conveys, interprets and extends the cultural connotations of advertising content to enhance the cultural attributes of advertising content, but also causes the psychological response of target audience in order to arouse the audience's recall and imagination closely associated with advertising content. Therefore, this paper conducts in combination with a number of examples a theoretical analysis on the relationship between music and advertising, features and types of music in advertising and research methods in psychology, discusses the questionnaire design patterns and demonstrates that advertising music producers should use their own knowledge of the relationship between music and advertising, features and types of music in advertising to select the most suitable questionnaire design pattern to develop a series of efficient and effective questionnaire for music in advertising.
\end{abstract}

Keywords-music in advertising; features; types; questionnaire design pattern

\section{INTRODUCTION}

The rapid development of science and technology promotes the ever-changing development of new media, and global multicultural collision impacts on the program patterns and contents of new media. Music in advertising, serving as an important part of new media's program patterns, is the music integrated in an advertisement to enhance the audience's advertising memorability with the involvement of sensory stimuli by constantly exploring the new connotations of soundcentered auditory element, and thus to cause the bond between the advertising content and the products for efficient product promotion. Music psychology is a field of research with practical relevance for many areas, including human musicality, auditory and musical perception, tone and music memorability, musical perception, psychological induction to music and audio stimulus. Researchers conducted empirical research (by using experiments, surveys and measurement methods) on samples in a scientific manner to get a scientific, valid and reliable research results, and by incorporating such research results, music in advertising helps achieve the overall promotional goals and even goes beyond the promotional goal of advertising content.

\section{INTERACTION OF MUSIC AND ADVERTISING AND ADVERTISING ELEMENTS}

\section{A. Music and Advertising}

The reason why music becomes one of the most important advertising elements is that: 1 . music helps enhance the advertising content as music can mirror lifestyle in an indirect and non-deterministic manner; 2. music can express emotions directly to motivate the target audience's emotional response when incorporated in advertisements; 3 . music can evoke the target audience's special emotions by revealing the era content; 4. music has the function of narration and descriptiveness to encourage the target audience's association and imagination by using acoustic stimulation and even to go beyond the promotional goals; 5 . music helps put the national culture in a special position by cuing the national characters to produce the target audience's recognition. Thus it can be seen that music plays a vital role in advertising campaigns because of such obvious cultural characteristics.

\section{B. Elements of Advertising}

The elements of advertising mainly refer to auditory and visual elements when classified based on sensory characteristic. Commonly, auditory element means language, music and sound, and the visual element refers to image and subtitles. An ad copy may incorporate either of such elements or both of such elements as the case may be. The language of advertising, known for its accuracy and strategically summarizing the advertising content, is mainly composed of narration and advertising actors' lines. A representative example is the language used in the advertising of Robust Pure Drinking Water, saying that 27-step purification process for enhanced purity and guaranteed quality. In a word, the various elements for aiding a television advertisement should have their respective common property and individuality for mutual complementation, among which, music element is a particularly important one. 


\section{THE CHARACTERISTICS AND TYPES OF MUSIC IN ADVERTISING}

\section{A. Characteristics of Music In Advertising}

Advertising is a compound cultural program pattern constituted with auditory and visual elements. The soundbased auditory element has a special functional attribute, and a extensive and comprehensive feature. On the one hand, a particular sound stimuli can intensify better the distinctive image and specific characteristics of the advertisements to have a powerful attraction, on the other hand, the incorporation of distinctive melody, tempo, timbre and musical instruments in the advertisements contributes to advertising communication and makes an advertisement more memorable to its target audience. Besides, the powerful attachment and integration of music with culture give the advertisements more profound connotations.

It is widely believed that music in advertising has the following characteristics: i. distinctive music style. The music integrated in an advertisement should strategically match with the style and content of the advertisement to its best. Therefore, music style has an exclusive cultural function. Music style, with its distinctive character, can be coupled with cultural content to achieve the purpose of cultural integration as much as possible, and be linked with innovative orchestration and gorgeous chords to lead the viewers to have a new viewpoint of advertising content. On the one hand, the unique cultural attribute of music style embodies the public's demand for musical culture and represents the popular melody and simple tonality to promote advertising content, for example, the introduction of major and minor modes and tonality from western music. On the other hand, the music style can display typical, particularly distinctive cultural style of folk music based on the common attachments to natural culture, for example, folk songs and instrumental music using the traditional Chinese pentatonic scales. ii. The diverse and compound elements of music in morphology. Flexible and changing musical structure and compositive and diverse rhythm is another notable characteristic of music in advertising, for example: for the musical form, it uses rhyme -repeated form, differently-rhymed form, special rhythmic pattern and other flexible and diversified structures; for the rhythm strategies, it uses the most common simple time signature, including ${ }^{\frac{2}{4}}, \frac{3}{4}, \frac{4}{4}$ and $\frac{1}{6}$ note and other various combinations of such time signatures; examples of complex time signatures including syncopation, upbeat, ${ }^{\frac{3}{8}}, \frac{6}{8}$ and triple note; it also uses mixed meter and variant meter for complex musical pattern.

\section{B. Types of Music in Advertising}

The music in advertising can be classified into vocal music and instrumental music by the types of representation, and lyrical music, narrative music, comedy music and sound used to create a typical and special atmosphere by the role of music in advertising. Generally, music in advertising can use only a single type, or integrates a variety of types.
Vocal music in advertising commonly uses vivid, simple and easily-understood lyrics coupled with appropriate melody. Some scholars believe that the original form of vocal music in advertising may resemble Recitative used in opera, and to be specific, the vocal music may derive from loudly vocal utterance for peddling or shouts delivered in an exaggerated way to emphasize the promotional content and then to draw the target audience's attention to make the advertising memorable to them, finally to obtain the promotional effect. Below gives a typical example:

Music in a series of Wahaha advertisements

$$
66 \searrow 535 \cdot 1
$$

\section{Wa Ha Ha Guo Nai}

Instrumental music in advertising generally uses these instrumental musical works famous at home and abroad. It employs typical and special melody, links with cultural connotations the music conveys with reference to the content, and gives full play to its complementary, lyrical and descriptive functions to arouse the target audience's association, and therefore to achieve the twofold promotional effect with less efforts, an example is the use of a regular meter of 52515 by Intel Company.

Music in advertising, no matter being produced in vocal music or instrumental music, or in other compound music types, can match advertising content by employing these classic musical elements of vocal music and instrumental music at domestic and abroad and sound cued with special atmosphere. For example, China Ping An Insurance Company uses the vocal musical piece named "Fare You Well" and BMW Group selects the instrumental musical works known as "Blue Danube". As the Father of Advertising, David Ogilvy, mentioned that a manufacturer who desire to use advertising to develop its brand image should fully and elaborately demonstrate the characters of the brand content, only in this way can the manufacturer capture the biggest market and make the maximized profit. An example is the broadcast advertising in relation to "SANTORY" Whiskey of Japanese sushi restaurant which won the best award in the 8th Folk Television Conference of Japan.

\section{Music content: Chopin's works, streams, birdsong.}

Interpretation: Life is short but art will last. Classical works can surely stand the test of time, and the whiskey produced with excellent creativity can likewise stand the test of time. The globally famous "SANTORY" whiskey with a 60 -year tradition is produced in Yamazaki, a place mostly suitable for wine brewing in Japan. The transparent liquor "sleeps" (is stored) in barrels day and night for a period of ten years, twenty years or thirty years, and as time goes on, the wine become fully matured and well-favored.

Sound elements: a clear sound as the wooden barrel is opened.

Interpretation: Hello! Everyone, the barrels are opened and the room is filled with the fragrance of "SANTORY". Look! The liquor shines like amber. The aged liquor is well-matured as classical music. 
Sound element: We hear the liquor gurgling when poured into a glass and the ice cube jingles when falling down the glass, and at the same time, smoothing music with pastoral color gently plays.

Interpretation: The best mate for you now is a glass of world-renowned iced "SANTORY" and a piece of worldknown music to immerse yourself in a wonderful world.

As can be seen from the sound elements and interpretation of "SANTORY" broadcast advertising, the advertisement repeatedly uses the typical sounds, including a clear sound heard when the barrel is opened, gurgle of wine when poured into a glass, jingle ice cube to stress the fully-matured and extra-mellowing "SANTORY" whiskey and to further emphasize the content features. The auditory stimulus provokes the audience's boundless association and embodies the color, smell and shape of language such that the audience can be immersed in the scene in person where they are holding a glass of iced world-known "SANTORY" Whiskey. Above all, Chopin's music with pastoral color drifts through the advertisement. The incorporation of classical elements of Chopin's musical works, including melody, rhythm, harmony and audio, are employed as a method of representation to embody the physical property of "SANTORY" Whiskey and explores profound cultural connotations of "SANTORY", meanwhile, it gives more profound cultural connotations to the individualized content of "SANTORY" advertisement to get more better promotional effect based on a comparison between fully-matured wine and the fragrance of Chopin's music.

\section{Music IN ADVERTISING AND RESEARCH METHOD IN PSYCHOLOGY - QUESTIONNAIRE DESIGN PATTERN}

As defined in a traditional American dictionary, a question (namely, questionnaire) is a research instrument consisting of a series of questions for the purpose of gathering information from respondents and especially designed for statistical analysis of the responses. A questionnaire consists of a number of questions that the respondent has to answer in a set format in person or in other ways for the purpose of gathering research information and data from the respondents. A questionnaire has the following characteristics: firstly, standardized format; secondly, a variety of types of questions and a wide range of application, less susceptible to objective limitations on research conditions; thirdly, pre-designed questionnaire items, making it easy to find out the cause-andeffect relationship of things; fourthly, standardized answers, making it simple to compile and analyze data on the computer.

\section{A. Structured Questionnaire (Close-ended Question)}

A structured questionnaire consists of a series of questions with a given number of options designed based on the researchers' research objectives and has the respondent pick an answer from such given options. Several types of response scales for structured questionnaire are distinguished: (1). dichotomous, where the respondent has two options, i.e. YES and No, AGREE and DISAGREE, requiring the respondent to only pick one option, an example is A Research of the Target Audience's Independent Choice of Music in Advertising involving the following questions: Q1: Is it possible for others to influence your choice of how to feel the music in advertising? A. Yes B. No; Q2: Do you view advertisements everyday without interruption? A Yes B No. (2). Paralleled, where the respondent has one or more than one answers from a number of options. The paralleled options can be bound or non-bound to the question. Bound question allows the respondent to pick only one or more than one options and non-bound question has open answers without any limitation for the respondent. For example: Q1 (bound question): Which area do you think music in advertising shall focus on? (please pick three options at most) A. practical content B. Informative C. Aesthetic function D. Additional functions E. Concerned the target audience's interest F. Cultural function G. Others Q2 (non-bound question): What kind of new media devices available for you to view music in advertising at home? (you may pick multiple options) A. IPTV B 4G phone C Tablet computer D. 3G phone E Others F. Nothing. (3) Ordered, where the respondent is required to logically pick his options from a given number of options from the most important to the least important. For example: What do you think is the major issues in the existing music in advertising? A. Low production standards B. Insufficient emphasis C. Outdated and stereotyped content D. Mismatch the advertising content E. Less connotation, and more superficial content F. I have no idea (Please pick three options from the most important to least important). (4) Hierarchical: where the respondent is allowed only to give one answer from a given number of options ranked in order. For example: Do you want to learn music in advertising? A. Yes B. No C. Yes, but I'm shy D. It doesn't matter. (5) Format-based, where the respondent has to pick only one corresponding option for each question in a set format. For example: Summary of Music in Advertising based on Viewing Time and Content. (6) Continuous, where the respondent is presented with another question if he picks one given option. For example: Do you sing a piece of music in advertising in a public place? A. Yes B No (if it is NO, please pick the reason) - a. There is no suitable music in advertising b. Forgot the melody and lyrics c. not interested.

\section{B. Semi-closed Questionnaire}

Typically, a semi-closed questionnaire adds one openended option to its given number of options to allow the respondent to pick if he fails to pick one option according to the actual situation. For example: The major reason why you pay attention to the music in advertising is to A. improve artistic cultivation B. grow up to be a producer of music in advertising C. entertainment and leisure D. Others.

\section{Open-ended Questionnaire}

An open-ended questionnaire, also known as nonstructured questionnaire, asks the respondent to formulate his own answer because of it setting open question and nonstandardized answer, allowing the respondent to answer the question completely depending on his understanding of the problems and his own actual situation. For this, the answer to an open-ended question is more likely to mirror the respondent's attitudes and point of view towards the problem and his psychological characteristics, such that the researchers can obtain detailed, rich and more valuable materials than the problem itself. However, an open-ended questionnaire has 
typical open-ended questions and objective answers, making it difficult to compile the data collected from the respondents. An example is the Research of Interest of Students Majored in Advertising in Courses of Music of Advertising, requiring the respondent to answer in the least words what most makes the respondent happy and what most frustrates the respondent in the process of learning music in advertising.

\section{Compound Questionnaire}

A compound questionnaire is the integration of structured and non-structured questionnaires. Mostly, a questionnaire focuses on closed-ended question and engages open-ended question as a supplement. A close-ended question generally has the questionnaire items for which the researchers is knowledgeable and can list a number of possible answers, but in contrast, an open-ended question typically involves these questionnaire items for which the researchers is less knowledgeable and fails to list a number of possible answers. For a majority of questionnaires, researchers commonly use the compound questionnaire.

\section{CONCLUSION}

As questionnaire is a simple-to-use research instrument for the research of music in advertising, most people are willing to use it. However, people tend to pay less attention to the relationship between music and advertising, characteristics and types of music in advertising, have no knowledge of the requirements or skills needed for questionnaire compilation, the selection of respondents and result analysis. They commonly design the questions at their own option and roughly analyze the gathered data to draw a conclusion, all of which weaken the efficiency and reliability of the research and accuracy of the research result, and consequently affect the production quality of music in advertising. Therefore, it is essential to involve the knowledge relevant to relationship of music and advertising, characteristics and types of music in advertising and the research methods in psychology in the selection of questionnaire design pattern for the purpose of developing an efficient and effective questionnaire for the music in advertising.

\section{REFERENCES}

[1] Zhu Qiuhua. A Brief Introduction of Music in Advertising [J]. People's Music, 1994, (08).

[2] Chen Bin, Cheng Jin. Music for Television and Films [M] Hangzhou: Zhejiang University Press, 2004.102-120.

[3] Zhou Shibin. Music Education and Research Methods in Psychology [M]. Shanghai: Shanghai Music Publishing House, 2006.23-28.

[4] Wu Wenhan. The Exploration and Analysis of Types of Music in Advertising and Psychological Reception Mechanism for the Brand Image Building [J]. Press Cycles, 2011, (3). 